\title{
Decreased sphingolipid synthesis in children with 17q21 asthma-risk genotypes
}

\author{
Jennie G. Ono, ${ }^{1}$ Benjamin I. Kim, ${ }^{2}$ Yize Zhao, ${ }^{3}$ Paul J. Christos, ${ }^{4}$ Yohannes Tesfaigzi, ${ }^{5}$ Tilla S. Worgall, ${ }^{2}$ and Stefan Worgall ${ }^{1,6}$ \\ 'Department of Pediatrics, Weill Cornell Medicine, New York, New York, USA. ²Department of Pathology and Cell Biology, Columbia University, New York, New York, USA. ${ }^{3}$ Department of Biostatistics, Yale \\ School of Public Health, New Haven, Connecticut, USA. ${ }^{4}$ Department of Healthcare Policy and Research, Weill Cornell Medicine, New York, New York, USA. ${ }^{5}$ Department of Pulmonary and Critical Care \\ Medicine, Harvard Medical School, Boston, Massachusetts, USA. ${ }^{6}$ Department of Cenetic Medicine, Weill Cornell Medicine, New York, New York, USA.
}

Risk for childhood asthma is conferred by alleles within the 17q21 locus affecting ORMDL sphingolipid biosynthesis regulator 3 (ORMDL3) expression. ORMDL3 inhibits sphingolipid de novo synthesis. Although the effects of 17q21 genotypes on sphingolipid synthesis in human asthma remain unclear, both decreased sphingolipid synthesis and ORMDL3 overexpression are linked to airway hyperreactivity. To characterize the relationship of genetic asthma susceptibility with sphingolipid synthesis, we analyzed asthma-associated 17q21 genotypes (rs7216389, rs8076131, rs4065275, rs12603332, and rs8067378) in both children with asthma and those without asthma, quantified plasma and whole-blood sphingolipids, and assessed sphingolipid de novo synthesis in peripheral blood cells by measuring the incorporation of stable isotopelabeled serine (substrate) into sphinganine and sphinganine-1-phosphate. Whole-blood dihydroceramides and ceramides were decreased in subjects with the 17q21 asthma-risk alleles rs7216389 and rs8076131. Children with nonallergic asthma had lower dihydroceramides, ceramides, and sphingomyelins than did controls. Children with allergic asthma had higher dihydroceramides, ceramides, and sphingomyelins compared with children with nonallergic asthma. Additionally, de novo sphingolipid synthesis was lower in children with asthma compared with controls. These findings connect genetic 17q21 variations that are associated with asthma risk and higher ORMDL3 expression to lower sphingolipid synthesis in humans. Altered sphingolipid synthesis may therefore be a critical factor in asthma pathogenesis and may guide the development of future therapeutics.

\section{Introduction}

Asthma in children is characterized by airway hyperreactivity and a strong genetic predisposition. The asthma-associated susceptibility locus 17q21 is strongly linked to childhood-onset asthma (1), and asthma-associated genotypes in this region correlate with the expression of ORMDL sphingolipid biosynthesis regulator 3 (ORMDL3) (1-5). Risk alleles within the 17q21 region that increase ORMDL3 are not only linked to childhood-onset asthma but also to wheezing during infancy and viral infections $(6,7)$.

ORMDL3 inhibits serine-palmitoyltransferase (SPT), the rate-limiting enzyme complex of de novo sphingolipid synthesis (Supplemental Figure 1; supplemental material available online with this article; https://doi.org/10.1172/JCI130860DS1) (8-10). Sphingolipids are essential building blocks of cellular membranes and constitute key cellular signaling mediators (11). SPT condensates an amino acid (usually serine) and a fatty acyl-CoA (usually palmitoyl-CoA) to produce 3-ketosphinganine, which is immedi-

\section{Related Commentary: p. 604}

Authorship note: TSW and SW contributed equally to this work. Conflict of interest: The authors have declared that no conflict of interest exists. Copyright: (C) 2020, American Society for Clinical Investigation.

Submitted: June 10, 2019; Accepted: October 31, 2019; Published: January 13, 2020. Reference information: / Clin Invest. 2020;130(2):921-926.

https://doi.org/10.1172/JCI130860. ately reduced to sphinganine. Sphinganine can be phosphorylated to sphinganine-1-phosphate or acylated to dihydroceramides. These analytes characterize the de novo sphingolipid synthesis pathway and are not generated through other means (8-10). Dihydroceramides are metabolized to ceramides that, together with sphingomyelins and complex glycosphingolipids, constitute the bulk of mammalian sphingolipids. Ceramides, sphingosine, sphingosine-1-phosphate (S1P), and complex sphingolipids are mainly generated in the recycling pathway and do not solely depend on SPT (9).

Experimentally, ORMDL3 overexpression and SPT inhibition cause airway hyperreactivity $(12,13)$, and mice transgenic for human ORMDL3 have hyperreactive airways and reduced serum and lung sphingolipids $(12,14)$. Pharmacologic inhibition or genetic impairment of SPT results in airway hyperreactivity without inflammation and in the absence of allergic sensitization (13). In humans, asthma (15-17) has mostly been associated with increased serum ceramides and S1P, as have other inflammatory or autoimmune diseases $(18,19)$. Elevated serum dihydroceramides distinguish exercise-induced bronchospasm, an asthma phenotype not typically associated with allergy, from other asthma endotypes (15). It remains unknown whether 17q21 asthma-associated genotypes affect sphingolipid synthesis or whether decreased sphingolipid synthesis (as would be predicted by the ORMDL3-associated $17 \mathrm{q} 21$ asthma genotypes) is a feature of childhood asthma. 

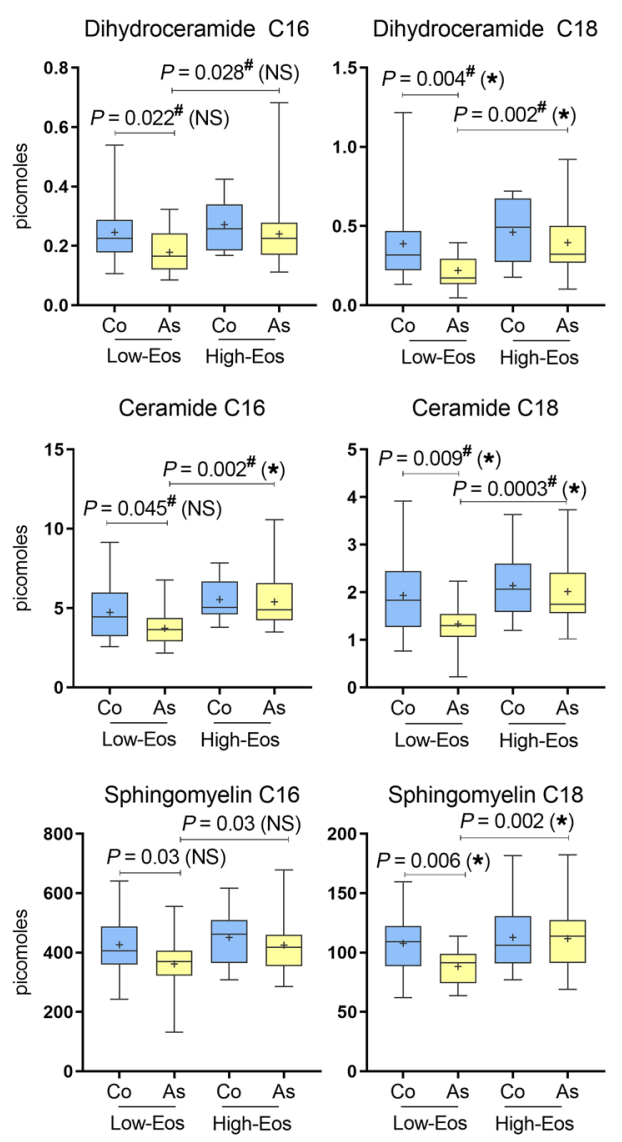
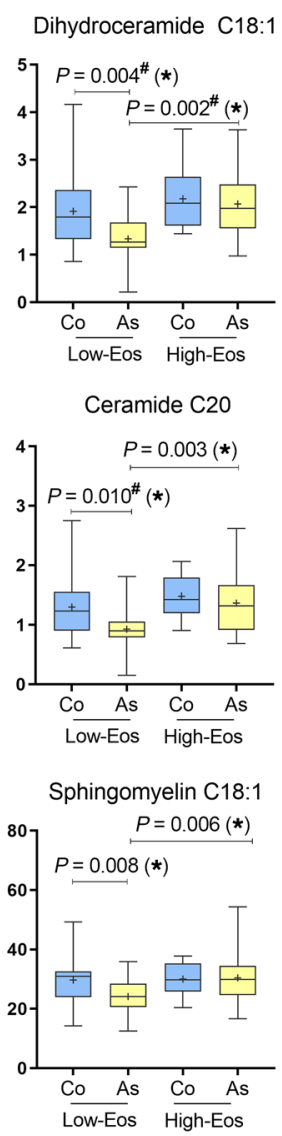
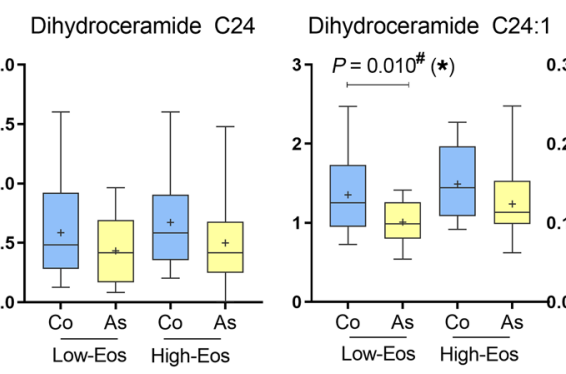

Sphinganine
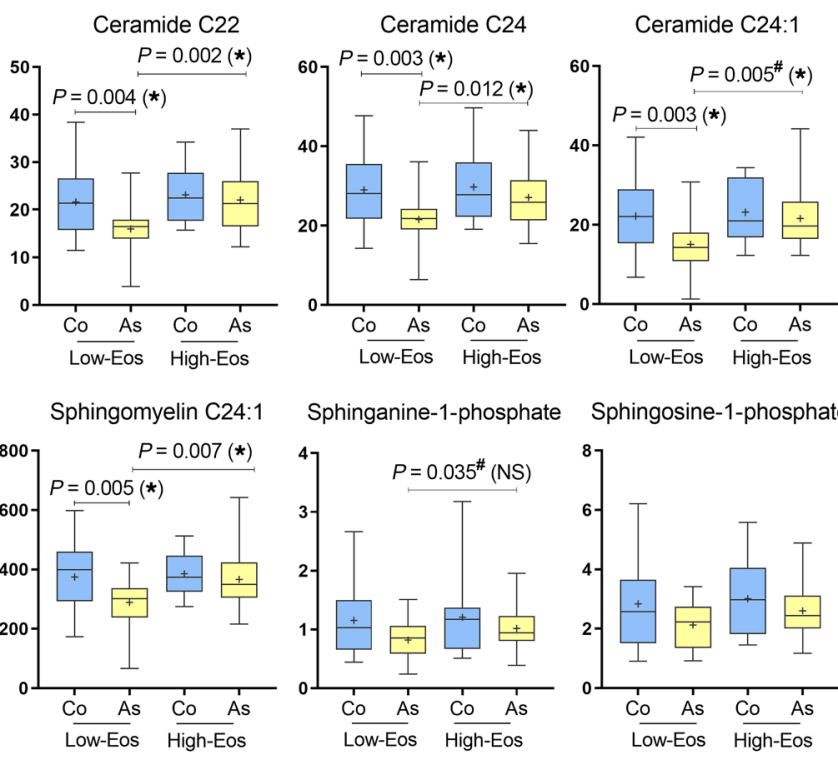

Sphinganine-1-phosphate

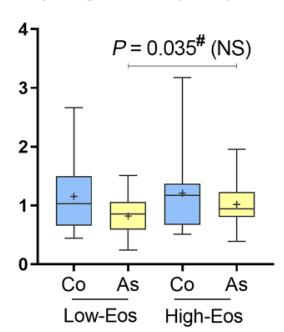

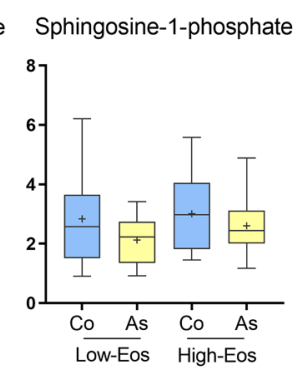

Figure 1. Sphingolipid composition in whole blood. Sphingolipids in control subjects (Co) and subjects with asthma (As), stratified by allergy group. Boxes represent the interquartile ranges with the median, and whiskers indicate the range. Low eosinophil counts (Low-Eos): $<300 / \mathrm{mL}$, high eosinophil counts (High-Eos): $\geq 300 / \mathrm{mL}$ in peripheral blood. The means are indicated by a plus sign and were compared by 2-sample $t$ test for normal data; for non-normal data, comparison of the median values is indicated by a pound sign. The corresponding $P$ values are reported if they were less than 0.05 . Significant $P$ values following the BH procedure (FDR $=0.05)$ are indicated by an asterisk if they fell below the BH-critical value of 0.014 . Controls with low-eosinophil counts ( $n=43$ ), asthma subjects with low-eosinophil counts $(n=16)$; controls with high-eosinophil counts $(n=14)$, asthma subjects with high-eosinophil counts ( $n=34)$.

To assess whether sphingolipid synthesis is altered in asthma and with 17q21 asthma-associated genotypes, we measured de novo synthesis in peripheral blood cells using stable isotope-labeled serine and determined genotypes at five 17q21 asthma-associated SNPs in children with and without asthma. Secondary objectives were to determine whether differences in blood sphingolipids are associated with variation at 17q21 asthma-associated SNPs and are altered in children with asthma with or without allergic markers.

\section{Results and Discussion}

In total, 120 subjects with asthma $(n=59)$ and without asthma $(n=61)$ between the ages of 5 and 17 years were enrolled in the study. Age and sex distributions were similar between groups (Supplemental Tables 1 and 2). Compared with controls, the subjects with asthma were more likely to have a history of atopy and allergies and had higher total IgE and eosinophils in peripheral blood. In our study, $70.5 \%$ of subjects with asthma reported being prescribed daily inhaled corticosteroid therapy, and $74 \%$ of the subjects with asthma received oral steroids at least once within the year of enrollment (Supplemental Table 2). The number of subjects with comorbid chronic medical conditions including obesity was similar between groups.
Whole-blood sphingolipids are decreased in nonallergic asthma. Sphingolipids were quantified in the plasma and whole blood of subjects with and without asthma and were stratified for the allergic group based on eosinophil count prior to analysis. In plasma, subjects with high-eosinophil-count-associated asthma ( $\geq 300$ eosinophils/mL) had increased dihydroceramide-C18 and ceramide-C2O compared with control subjects with high-eosinophil counts (Supplemental Figure 2). Sphingolipids did not differ between subjects with asthma and controls with low eosinophil counts (<300 eosinophils/mL) (Supplemental Figure 2). In contrast, sphingolipid profiles in whole blood showed lower dihydroceramides (C18, C18:1, C24:1), ceramides (C18, C20, C22, C24, C24:1), and sphingomyelins (SM18, SM18:1, SM24:1) in asthma subjects with low-eosinophil counts compared with controls with low-eosinophil counts (Figure 1). Whole-blood sphingolipid levels between asthma subjects with high-eosinophil counts and controls with high-eosinophil counts were similar (Figure 1).

In high-eosinophilic asthma, dihydroceramides (C18, C18:1), ceramides (C16, C18, C20, C22, C24, C24:1), and sphingomyelins (SM18, SM18:1, SM24:1) were increased compared with low-eosinophilic asthma (Figure 1). We detected no differences in plasma or whole-blood sphingolipids between control sub- 

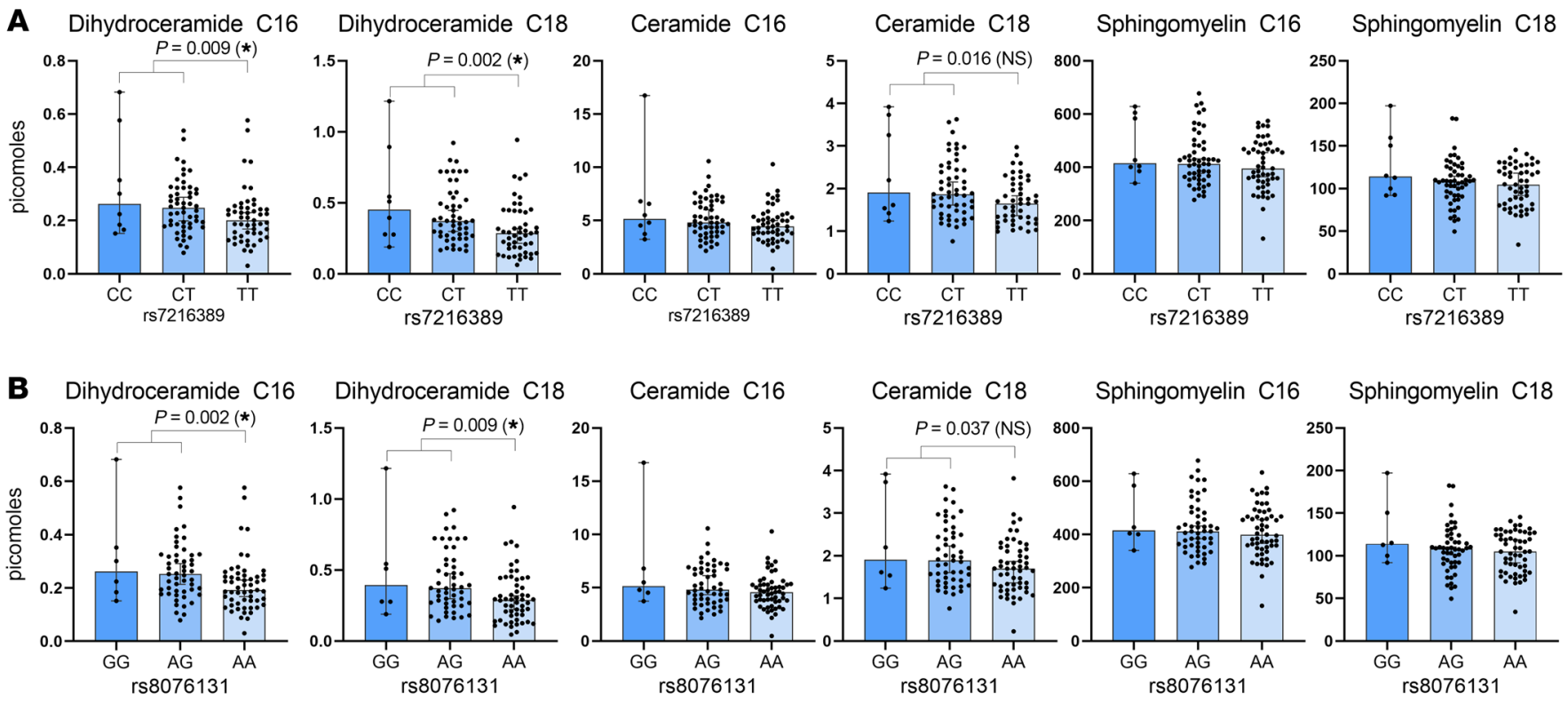

Sphingomyelin C18

Figure 2. Whole-blood sphingolipids with an acyl-chain length of C16-18 in all subjects. Examples of sphingolipid masses in combined asthma and control subjects based on genotype at (A) rs7216389 ( $n=8 \mathrm{CC} ; n=53 \mathrm{CT} ; n=51$ TT) and (B) rs8076131 ( $n=6$ GG; $n=51$ AC; $n=55$ AA). In total, 18 individual sphingolipids were measured for each genotype. Individual values are shown; bars represent the median with a $95 \% \mathrm{Cl}$, compared by Mann-Whitney $U$ test. Significant $P$ values following adjustment for a FDR of 0.05 are indicated with an asterisk, as they fell below the BH-critical value of 0.0092 .

jects with high eosinophil counts and those with low eosinophil counts (Figure 1 and Supplemental Figure 2), nor were differences detected in whole blood when stratified for IgE levels (Supplemental Figure 3). Overall, these results demonstrate altered sphingolipids in the plasma and whole blood of children with asthma, with higher levels in plasma and lower levels in whole blood, and highlight a potential effect of allergy on sphingolipids in asthma. The more pronounced decrease in whole-blood sphingolipids in nonallergic compared with allergic asthma may either reflect a limited study size or that other factors affect sphingolipid levels in allergic asthma. The latter could further explain the strong association of 17q21 asthma-risk genotypes with nonallergic asthma described in the original GWAS (1) and in various studies since $(4,20)$. The increase in whole-blood dihydroceramides, ceramides, and sphingomyelins in allergic compared with nonallergic asthma, which was not observed in controls, suggests an additional effect of allergic sensitization on sphingolipid metabolism in asthma that needs further investigation.

Elevated levels of serum or plasma sphingolipids, mainly S1P, dihydroceramides, and ceramides, have been described in children with exercise-associated wheezing (15) and in small groups of adults with asthma $(16,17)$. The increased levels of plasma dihydroceramide-C18 and ceramide-C20 in asthma in the current study are consistent with a previous observation of higher serum dihydroceramide-C18 in children with exercise-associated wheezing (15). A key finding of the current study is that plasma and whole-blood sphingolipids are fundamentally different. Since whole blood contains abundant erythrocytes and leukocytes absent in plasma, it is likely that the lower sphingolipid levels in nonallergic asthma reflect decreased membrane-associated sphingolipid levels. Whether this difference in sphingolipid levels reflects structural alterations in blood cell membranes that contribute to asthma susceptibility and airway hyperreactivity or is instead a marker of a perturbed system cannot be answered with this study.

$17 q 21$ SNPs are associated with decreased dihydroceramide and ceramides. To evaluate the relationship between genetic variation at 17q21 and sphingolipids in whole blood, we determined genotypes at 5 asthma-associated 17q21 SNPs (rs7216389, rs8067378, rs4065275, rs8076131, and rs12603332). rs7216389 (risk allele T) was the lead SNP identified in the first GWAS (1) and an expression quantitative trait loci (eQTL) for ORMDL3 in the lung (21) and whole blood (4, 22). Rs8067378 (risk allele A) has been associated with asthma and expression of GSDMB, but not ORMDL3, in bronchial epithelial cells (23). Rs4065275 (risk allele G) and rs8076131 (risk allele A) are associated with

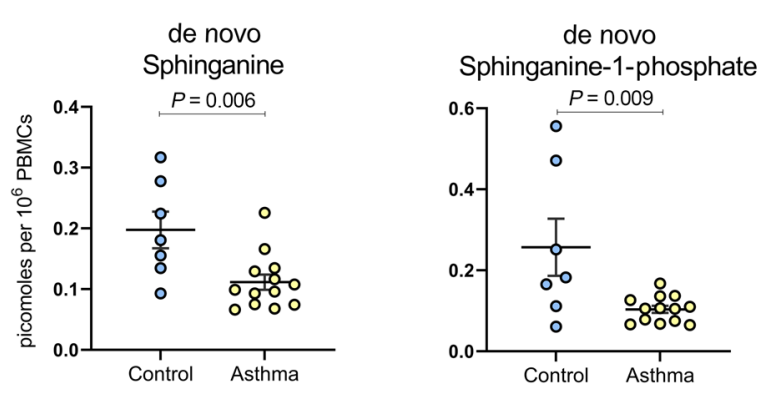

Figure 3. De novo sphingolipid synthesis is decreased in children with asthma. Newly synthesized sphinganine and sphinganine-1-phosphate in control subjects $(n=7)$ and subjects with asthma $(n=13)$ following metabolic labeling of the substrate $\left(C^{13} \mathrm{~N}^{15}\right.$-serine) in PBMCs. Individual values are shown, and the means were compared using a 2-sample $t$ test. 
A
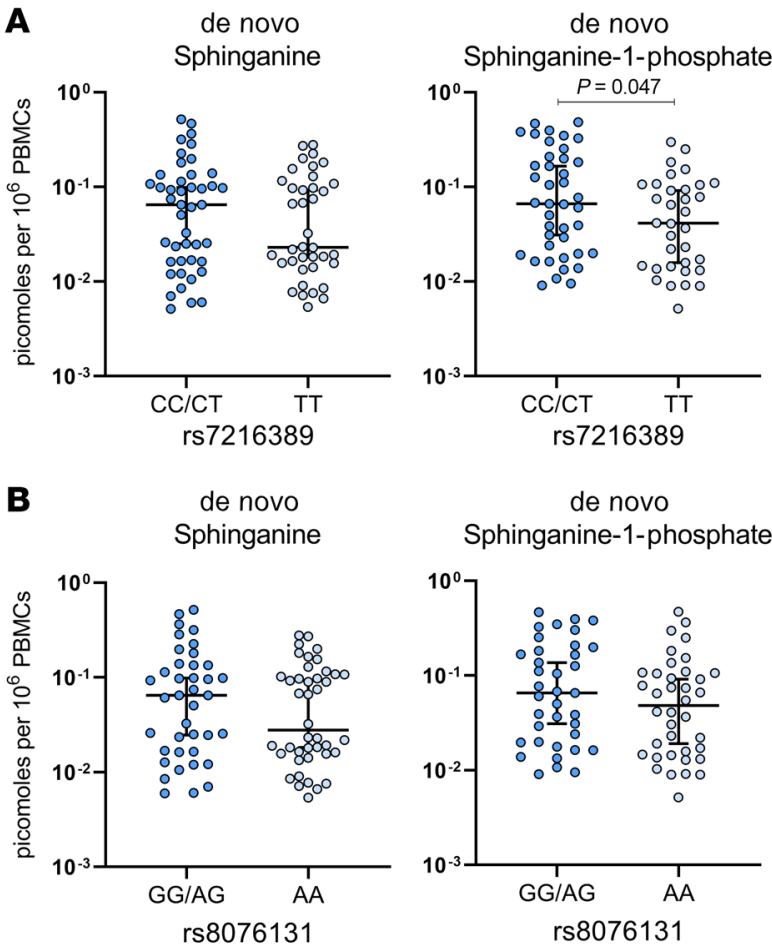

increased asthma susceptibility $(4,24)$ and ORMDL3 expression in lung and whole blood (25). Rs12603332 (risk allele C) is the lead SNP for asthma in Mexican Americans and African Americans (26) and methylation (me)QTL/eQTL for ORMDL3 and GSDMB in blood cells $(2,4)$. Individual genotype frequencies are presented in Supplemental Results and Supplemental Table 3. Asthma-risk alleles for both rs7216389 (risk allele T) and rs8076131 (risk allele A) correlated with a decrease in 4 dihydroceramides (C16, C18, C24, and C24:1) and 3 ceramides (C16, C18, and C20); rs7216389 was additionally associated with decreased ceramide-C18:1 (Supplemental Table 4). Risk alleles of the asthma-associated genotypes rs8067378, rs4065275, and rs12603332 had no correlation with the measured whole-blood sphingolipids. Following stratification only for subjects with asthma, rs7216389 remained associated with 2 dihydroceramides (C16 and C18) and rs8076131 with 4 dihydroceramides (C16, C18, C24, and C24:1) and 3 ceramides (C16, C18, and C2O) (Supplemental Table 5).

We next analyzed the association of rs7216389 and rs8076131 genotypes with whole-blood sphingolipids of all subjects and observed decreased sphingolipids in a genotype-dependent manner (Figure 2, A and B). Specifically, for rs7216389, mean levels of four dihydroceramides (C16, C18, $\mathrm{C} 24$, and C24:1), sphinganine-1-phosphate, and 3 ceramides (C16, C18, and C20) were decreased in an asthma-risk genotype-dependent manner $(\mathrm{TT}<\mathrm{CT}<\mathrm{CC})$ (Supplemental Table 6). For rs8076131, four dihydroceramides (C16, C18, C24, and $\mathrm{C} 24: 1)$ and 3 ceramides (C16, C18, and C20) decreased in an asthma-risk genotype-dependent manner $(\mathrm{AA}<\mathrm{GA}<\mathrm{GG}$ ) (Supplemental Table 7). Sphinganine, sphingosine, S1P, and sphingomyelins were not associated with any of the SNP genotypes (Supplemental Tables 6 and 7). These data suggest that 17q21
Figure 4. De novo sphingolipid synthesis is decreased in children with 17q21 asthma-risk genotypes. Newly synthesized sphinganine ( $\mathrm{Sa}$ ) and sphinganine-1-phosphate (Sa1P) in PBMCs of all subjects, incubated with ${ }^{13} \mathrm{~N}^{15}$-serine and stratified by genotype (A) rs7216389 ( $n=44 \mathrm{Sa}$ CC/CT; $n=38 \mathrm{TT} ; n=41 \mathrm{Sa} 1 \mathrm{P} \mathrm{CC/CT;} n=39 \mathrm{TT}$ ) and (B) rs8076131 ( $n=$ $41 \mathrm{Sa} \mathrm{GG/AG;} n=42 \mathrm{TT} ; n=39 \mathrm{Sa1P}$ GG/AG; $n=38 \mathrm{AA}$ ). Individual data points are shown; bars represent the median and a $95 \% \mathrm{Cl}$, compared by Mann-Whitney $U$ test. (C) De novo synthesis of sphinganine and sphinganine-1-phosphate by rs7216389 genotype and stratified by group. Bars represent the median with a $95 \% \mathrm{Cl}$. Comparisons between asthma groups were done with a Kruskal-Wallis $\mathrm{H}$ test $[\mathrm{H}(4)=6.534, P=0.08]$.

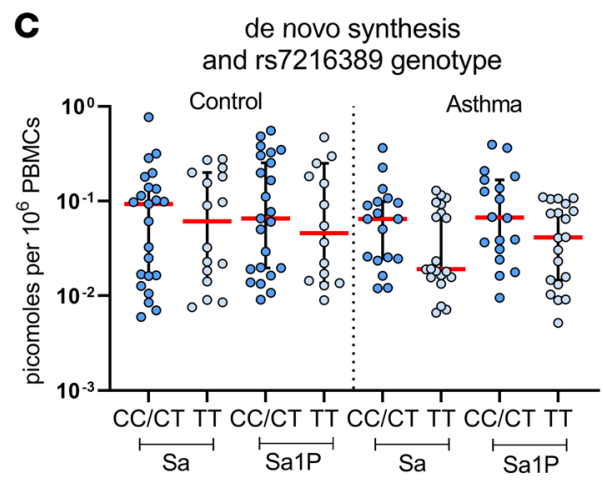

asthma-risk alleles are associated with decreased whole-blood sphingolipids. Of note, these results cannot be compared with a study involving a Swiss adult population, in which no associations were found between hydrolytic products of all plasma sphingolipids and rs7216389 genotypes (27). Those analyses cannot distinguish long-chain bases originating from de novo synthesis and were not performed in whole blood. It is of interest that rs7216389 and rs8076131 risk alleles may protect children from wheezing through dose-dependent exposure to farm animals, suggesting that these genotypes could constitute genetic risk as well as confer environmental protection (6).

Sphingolipid de novo synthesis is decreased in asthma and is associated with an asthma-risk genotype. Given the high expression of ORMDL3 in T and B cells (25), we used PBMCs to assess whether de novo sphingolipid synthesis is altered in children with asthma. PBMCs were incubated with stable isotopelabeled serine $\left(\mathrm{C}^{13} \mathrm{~N}^{15}\right)$, and the $[\mathrm{M}+3]$ isotopes for sphinganine and sphinganine-1-phosphate were quantified as the primary outcome. Stable isotope-labeled sphinganine and sphinganine-1-phosphate were lower in children with asthma compared with levels in controls $(P<0.05$; Figure 3$)$. These findings were replicated in a second analysis of PBMCs from additional control and asthma subjects (Supplemental Figure 4). When all data were combined and stratified by rs7218369 genotype, de novo-synthesized sphinganine and sphinganine-1-phosphate were lowest with the asthma-risk TT genotype $(\mathrm{CC}+\mathrm{CT}>\mathrm{TT}$; Figure 4A), and risk allele A (GG+AG>AA) for rs8076131 (Figure 4B). The rs7216389-TT genotype remained associated with lower de novo-synthesized sphinganine and sphinganine-1-phosphate in both control and asthma subjects following further stratification for each group, although this did not achieve statistical significance (Figure 4C). 
To assess whether the association between the rs7216389 asthma-risk genotype and lower sphingolipid synthesis is found in airway epithelial cells, we evaluated sphingolipids in adult primary epithelial cells with either rs7216389-TT or rs7216389-CC genotypes, grown as air-liquid interface cultures. This is an asthma-relevant cell type with high expression of ORMDL3 (28) and challenging to obtain from children. De novo-synthesized sphinganine and dihydroceramide-C24 were lower in cells with the risk-associated TT genotype compared with cells with the CC genotype. No differences were detected for the naturally occurring sphingolipids S1P, ceramide-C18, or sphingomyelin-C18 (Supplemental Figure 5). These results suggest that 17q21 genotypes that increase ORMDL3 expression are associated with decreased products of de novo sphingolipid synthesis.

The functional link of genetic variations in the 17q21 region to asthma has been mainly attributed to increased ORMDL3, although it remains unclear how this protein confers increased asthma risk (4). Although it is undisputed that ORMDL3 is a key regulator of sphingolipid synthesis, other direct or indirect effects of ORMDL3 on ER stress and inflammatory responses have been proposed as the mechanistic links to asthma pathogenesis $(20,29)$. Here, we demonstrate that altered sphingolipids are associated with childhood asthma, as we found decreased de novo sphingolipid synthesis in blood cells of children with asthma and an association of 17q21 region asthma-risk genotypes with lower measured sphingolipids and sphingolipid synthesis in whole blood.

A limitation of this study is that because sphingolipids cannot be measured noninvasively in the airways of children with asthma, the assessment therefore relies on indirect measurements of blood and blood cells. Our findings in cultured airway epithelial cells that showed decreased sphingolipids in cells carrying the asthma-risk genotype support the idea that 17q21 genotypes can affect sphingolipid composition in respiratory tract-relevant cells. In this study, we did not assess ORMDL3 expression. The findings of a direct correlation between mRNA expression of ORMDL3 or other asthma-associated 17q21 genes in PBMCs and asthma $(1,2,4)$ or asthma genotypes $(24)$ have been inconsistent, with genotype-dependent gene expression for the asthma-associated SNPs rs8076131 and rs4065275 seen only after stimulation of PBMCs.

Overall, this study provides the first direct link, to our knowledge, of asthma genetics to decreased sphingolipid synthesis in humans, suggesting that impaired de novo sphingolipid synthesis is a direct result of increased ORMDL3. Altered cellular sphingolipids might therefore be a factor in asthma pathogenesis, affecting airway reactivity. Whether this factor is conferred directly through sphingolipid-triggered mechanisms or through interactions with other ORMDL3-associated inflammatory effects remains to be elucidated.

\section{Methods}

Study design and enrollment of subjects. For this case-control study with prevalent cases, children (5-17 years of age) were recruited from 2 ambulatory sites in New York, New York, providing pediatric primary and pulmonology care. The children were classified as having asthma if they had a diagnosis of asthma by a pediatric pulmonologist and had recent symptoms (see Supplemental Methods for complete details).
All subjects underwent collection of blood for sphingolipid quantification, measurement of total IgE and eosinophil counts for characterization of allergic status, DNA isolation and genotyping at five 17q21 asthma-associated SNPs, and isolation of PBMCs for assessment of de novo sphingolipid synthesis.

Sphingolipid quantitation. Sphingolipids were quantified in plasma, whole blood, PBMCs, and airway epithelial cells using high-pressure liquid chromatography electrospray ionization tandem mass spectrometry (HPLC-MS/MS) with minor modification of a previously described method (30) (see Supplemental Methods for complete details).

SNP genotyping. SNP genotyping was performed using TaqMan SNP Genotyping Assays (see Supplemental Methods for complete details).

Metabolic labeling and measurement of de novo-synthesized sphinganine and sphinganine-1-phosphate. Sphingolipid de novo synthesis was measured in PBMCs isolated from whole blood of control and asthma subjects by metabolic labeling with the SPT substrate serine. This method can reliably assess SPT activity (31) and is fully described in the Supplemental Methods.

Statistics. A 2-sample $t$ test or $\chi^{2}$ with Fisher's exact test was used to compare 2 groups depending on the type of data. ANOVA and logistic regressions were used to perform univariable or multivariable association analyses. Nonparametric testing was performed where data were not normally distributed. A $P$ value of less than 0.05 was considered significant. To address multiple comparisons and control for the FDR, we applied the Benjamini-Hochberg (BH) procedure (32) to analyses involving 3 or more comparisons, using a maximum FDR of 0.05. Associations for SNPs and individual sphingolipids were analyzed using Pearson's correlation coefficient (see Supplemental Methods for complete details).

Study approval. The study was approved by the Weill Cornell IRB (protocol 1206012409) and the Western IRB of New York Hospital Queens (protocol 20150736). Written informed consent was obtained from all participants prior to inclusion in the study.

\section{Author contributions}

JGO conducted experiments and analyzed and interpreted the data. BIK performed data analysis and interpretation. YZ and PJC performed statistical analysis and review. YT conducted epithelial cell experiments. JGO, SW, and TSW conceptualized and designed the study, interpreted the data, and wrote the manuscript. All authors contributed to the drafting of the manuscript.

\section{Acknowledgments}

JGO and this study were supported by the National Center for Advancing Translational Sciences, NIH (KL2-TR000458 and UL1-TR00457). YZ and PJC are partially supported by the National Center for Advancing Translational Sciences, NIH (UL1TR002384-01). We acknowledge the generous support of Ronay and Richard Menschel, Christine and Pasco Alfaro, and Casey S. and Noah N. Weiss. We additionally acknowledge Rosalyn Chan for study coordination and recruitment of subjects and Whenzu Wu for technical expertise.

Address correspondence to: Stefan Worgall, Department of Pediatrics, Weill Cornell Medicine, 413 East 69th Street, Room 1200, New York, New York 10021, USA. Phone: 646.962.6236; Email: stw2006@med.cornell.edu. 
1. Moffatt MF, et al. Genetic variants regulating ORMDL3 expression contribute to the risk of childhood asthma. Nature. 2007;448(7152):470-473.

2. Acevedo N, et al. Risk of childhood asthma is associated with $\mathrm{CpG}$-site polymorphisms, regional DNA methylation and mRNA levels at the GSDMB/ORMDL3 locus. Hum Mol Genet. 2015;24(3):875-890.

3. Verlaan DJ, et al. Allele-specific chromatin remodeling in the ZPBP2/GSDMB/ORMDL3 locus associated with the risk of asthma and autoimmune disease. Am J Hum Genet. 2009;85(3):377-393.

4. Stein MM, et al. A decade of research on the 17q1221 asthma locus: piecing together the puzzle. JAllergy Clin Immunol. 2018;142(3):749-764.e3.

5. Lluis A, et al. Asthma-associated polymorphisms in 17q21 influence cord blood ORMDL3 and GSDMA gene expression and IL-17 secretion. JAllergy Clin Immunol. 2011;127(6):1587-94.e6.

6. Loss GJ, et al. The Early Development of Wheeze. Environmental Determinants and Genetic Susceptibility at 17q21. Am J Respir Crit Care Med. 2016;193(8):889-897.

7. Calışkan M, et al. Rhinovirus wheezing illness and genetic risk of childhood-onset asthma. N Engl J Med. 2013;368(15):1398-1407.

8. Davis D, Kannan M, Wattenberg B. Orm/ ORMDL proteins: gate guardians and master regulators. Adv Biol Regul. 2018;70:3-18.

9. Breslow DK, et al. Orm family proteins mediate sphingolipid homeostasis. Nature. 2010;463(7284):1048-1053.

10. Davis DL, Gable K, Suemitsu J, Dunn TM, Wattenberg BW. The ORMDL/Orm-serine palmitoyltransferase (SPT) complex is directly regulated by ceramide: reconstitution of SPT regulation in isolated membranes. J Biol Chem. 2019;294(13):5146-5156.

11. Hannun YA, Obeid LM. Principles of bioactive lipid signaling: lessons from sphingolipids. Nat Rev Mol Cell Biol. 2008;9(2):139-150.
12. Miller M, et al. ORMDL3 transgenic mice have increased airway remodeling and airway responsiveness characteristic of asthma. J Immunol. 2014;192(8):3475-3487.

13. Worgall TS, et al. Impaired sphingolipid synthesis in the respiratory tract induces airway hyperreactivity. Sci Transl Med. 2013;5(186):186ra67.

14. Miller M, Rosenthal P, Beppu A, Gordillo R, Broide DH. Oroscomucoid like protein 3 (ORMDL3) transgenic mice have reduced levels of sphingolipids including sphingosine-1-phosphate and ceramide. JAllergy Clin Immunol. 2017;139(4):1373-1376.e4.

15. Perzanowski MS, et al. Distinctserum sphingolipid profiles among school-aged children with exercise-induced wheeze and asthma persistence. Am J Respir Crit Care Med. 2017;195(8):1068-1070.

16. Kowal K, Żebrowska E, Chabowski A. Altered sphingolipid metabolism is associated with asthma phenotype in house dust mite-allergic patients. Allergy Asthma Immunol Res. 2019;11(3):330-342.

17. Oyeniran C, et al. Aberrant ORM (yeast)-like protein isoform 3 (ORMDL3) expression dysregulates ceramide homeostasis in cells and ceramide exacerbates allergic asthma in mice. J Allergy Clin Immunol. 2015;136(4):1035-46.e6.

18. Hla T, Dannenberg AJ. Sphingolipid signaling in metabolic disorders. Cell Metab. 2012;16(4):420-434.

19. Maceyka M, Spiegel S. Sphingolipid metabolites in inflammatory disease. Nature. 2014;510(7503):58-67.

20. Das S, Miller M, Broide DH. Chromosome 17q21 genes ORMDL3 and GSDMB in asthma and immune diseases. Adv Immunol. 2017;135:1-52.

21. Hao K, et al. Lung eQTLs to help reveal the molecular underpinnings of asthma. PLoS Genet. 2012;8(11):e1003029.

22. GTEx Consortium. Human genomics. The Genotype-Tissue Expression (GTEx) pilot analysis: multitissue gene regulation in humans. Science. 2015;348(6235):648-660.

23. LiX, et al. eQTL of bronchial epithelial cells and bronchial alveolar lavage deciphers GWAS-identified asthma genes. Allergy. 2015;70(10):1309-1318.

24. Schedel M, et al. Polymorphisms related to ORMDL3 are associated with asthma susceptibility, alterations in transcriptional regulation of ORMDL3, and changes in TH2 cytokine levels. J Allergy Clin Immunol. 2015;136(4):893-903.e14.

25. Schmiedel BJ, et al. 17q21 asthma-risk variants switch CTCF binding and regulate IL-2 production by T cells. Nat Commun. 2016;7:13426.

26. Galanter J, et al. ORMDL3 gene is associated with asthma in three ethnically diverse populations. Am JRespir Crit Care Med. 2008;177(11):1194-1200.

27. Zhakupova A, et al. ORMDL3 expression levels have no influence on the activity of serine palmitoyltransferase. FASEB J. 2016;30(12):4289-4300.

28. Löser S, et al. Pulmonary ORMDL3 is critical for induction of Alternaria-induced allergic airways disease. J Allergy Clin Immunol. 2017;139(5):1496-1507.e3.

29. Zhang Y, Willis-Owen SAG, Spiegel S, Lloyd CM, Moffatt MF, Cookson WOCM. The ORMDL3 asthma gene regulates ICAM1 and has multiple effects on cellular inflammation. Am J Respir Crit Care Med. 2019;199(4):478-488.

30. Bui HH, Leohr JK, Kuo MS. Analysis of sphingolipids in extracted human plasma using liquid chromatography electrospray ionization tandem mass spectrometry. Anal Biochem. 2012;423(2):187-194.

31. Ren J, et al. Quantification of 3-ketodihydrosphingosine using HPLC-ESI-MS/MS to study SPT activity in yeast Saccharomyces cerevisiae. J Lipid Res. 2018;59(1):162-170.

32. Glickman ME, Rao SR, Schultz MR. False discovery rate control is a recommended alternative to Bonferroni-type adjustments in health studies. JClin Epidemiol. 2014;67(8):850-857. 